\title{
Dynamic exciton localisation in a pyrene-BODIPY-pyrene dye conjugate
}

Nina Auerhammer, ${ }^{a}$ Alexander Schulz, ${ }^{a}$ Alexander Schmiedel, ${ }^{a}$ Marco Holzapfel, ${ }^{a}$ Joscha Hoche $^{\mathrm{b}}$, Merle I. S. Röhr, ${ }^{\mathrm{a}, \mathrm{b}}$, Roland Mitric, ${ }^{\mathrm{b} *}$ Christoph Lambert ${ }^{\mathrm{a} *}$

anstitute of Organic Chemistry, Center for Nanosystems Chemistry, Universität Würzburg

Am Hubland, D-97074 Würzburg, Germany

bInstitute of Physical and Theoretical Chemistry, Universität Würzburg

Am Hubland, D-97074 Würzburg, Germany

e-mail: christoph.lambert@uni-wuerzburg.de

\begin{abstract}
The photophysics of a molecular triad consisting of a BODIPY dye and two pyrene chromophores attached in 2-position are investigated by steady state and fs-time resolved transient absorption spectroscopy as well as by field induced surface hopping (FISH) simulations. While the steady state measurements indicate moderate chromophore interactions within the triad, the time resolved measurements show upon pyrene excitation a delocalised excited state which localises onto the BODIPY chromophore with a time constant of 0.12 ps. This could either be interpreted as an internal conversion process within the excitonically coupled chromophores or as an energy transfer from the pyrenes to the BODIPY dye. The analysis of FISH-trajectories reveals an oscillatory behaviour where the excitation hops between the pyrene units and the BODIPY dye several times until finally they become localised on the BODIPY chromophore within $100 \mathrm{fs}$. This is accompanied by an ultrafast nonradiative relaxation within the excitonic manifold mediated by the nonadiabatic coupling. Averaging over an ensemble of trajectories allowed us to simulate the electronic state population dynamics and determine the time constants for the nonradiative transitions that mediate the ultrafast energy transfer and exciton localisation on BODIPY.
\end{abstract}

\section{Introduction}

Whether an ensemble of two or more different chromophores is excited by light of certain energy as a whole or whether only one of these chromophores is excited and energy transfer to other chromophores occurs as a possible follow up process is one of the most 
fundamental issues in photophysics of complex chromophores. If the latter scenario applies, the rate of excitation energy transfer processes may span a range from several ns to several tens of fs. On the slower time scale an incoherent energy transfer from an excited energy donor to a farther apart situated energy acceptor often described by Förster's resonance energy transfer (FRET) is operative. Here, donor and acceptor are so weakly coupled that they retain the spectroscopic characteristics of the isolated components but other issues such as orientation of transition moments and spectral overlap of donor fluorescence with acceptor absorption are important parameters governing the rate of the process. ${ }^{1-8}$ On the other extreme, two or more chromophores may be coupled so strongly that the eigenstates of the newly formed super-chromophore deviate dramatically from those of its constituents. Often, these new eigenstates can be constructed using exciton coupling theory and energy transfer between the constituents is coherent until dephasing and relaxation take place..$^{9-15}$ The latter can be viewed as an internal conversion process. However, the intermediate coupling regime is also often found and has attracted much attention in recent years. ${ }^{16,17}$ Quite recently we addressed the exciton dynamics in a series of covalently linked squaraine dimers spanning the range from weak to intermediate to the strong coupling regime. ${ }^{18}$ In another work we described the optical properties of some dye conjugates consisting of transindolenine squaraine and BODIPY dyes linked by alkyne spacers. ${ }^{19}$ Those dye conjugates are in the strong coupling regime where the exciton coupling energy exceeds $1000 \mathrm{~cm}^{-1}$. In this paper, we concentrate on triad systems where a BODIPY chromophore is combined with two pyrene molecules attached via an alkyne spacer to each end of the chromophore. In principle, in this case the pyrene may act as the energy donor and the BODIPY dye as the acceptor. Here the eigenstates of the unperturbed chromophore constituents are energetically much farther apart as in the case of the BODIPY-squaraine conjugates mentioned above.

Pyrene was chosen as the formal energy donor as it possesses several special electronic properties making it an attractive chromophore for energy transfer purposes. Pyrene has a high molar extinction coefficient in the UV region and the absorption spectrum is dominated by the transition to the $S_{2}$ state (polarised along the long molecular axis (x), often called $L_{a}$ band) because the $S_{1}$ state ( $y$-polarised, $L_{b}$ band) is almost forbidden. ${ }^{20,}{ }^{21}$ Because internal conversion from $S_{2}$ to $S_{1}$ is ultrafast (75-85 fs), ${ }^{22,23}$ fluorescence is emitted from the $S_{1}$ state, leading to an exceptionally high apparent Stokes shift. Due to the low oscillator strength, the $\mathrm{S}_{1}$ lifetime is rather long (several hundred nanoseconds) which makes pyrene useful for nonradiant energy transfer. ${ }^{22,}{ }^{24}$ Keeping these properties in mind it is not surprising that pyrene has been used as a chromophore in numerous studies for optical applications. ${ }^{25}$ However, the above mentioned symmetry properties of pyrene excited states only may have an influence on photophysical processes if pyrene is attached to other energy acceptors via its 
2- or 7-position and the (local) symmetry is preserved. Binding pyrene at its 2- or 7-position also leaves the typical pyrene properties almost undisturbed because both HOMO and LUMO possess a nodal plane along the long molecular axis going through the 2- and 7position. However, if pyrene is substituted at the typically employed 1-position, the molecular symmetry is distorted. Even though the substitution of pyrene in 2- and 7-position has been facilitated by using Ir-catalysed reactions by Marder et al. a decade ago, only a few studies that use this substitution pattern are known to the best of our knowledge. ${ }^{26,27}$

To expand the photophysical studies of the 2,7-substituted pyrene chromophores we have synthesised the dye conjugate $\mathbf{P y}_{2} \mathbf{B}$ (see Fig. 1) to investigate the influence of the pyrene excited states on possible excitonic interactions with - or energy transfer to the BODIPY28 chromophore.

BODIPY ${ }^{29,}{ }^{30}$ dyes usually possess $C_{2 v}$ molecular symmetry (for molecular structure see Fig. 1Fig. 1) and display a high and rather narrow absorption band, distinctly red-shifted from pyrene, as well as high to medium fluorescence quantum yields. The lowest energy band is polarised perpendicular to the $C_{2}$ axis, that is, along the long molecular axis (x). For $S_{2}$ and $S_{3}$ the situation is less clear as they overlap strongly. One of those is polarised parallel to $S_{1}$ the other perpendicular. ${ }^{30}$

Especially the BODIPY chromophore is an ideal building block in energy transfer arrays from a synthetic as well as photophysical point of view. For this reason, it has extensively been used in energy transfer research because it can easily be substituted at various positions, it has a high absorption coefficient in the visible as well as a high fluorescence quantum yield and it shows a very low tendency to form triplet excited states. ${ }^{31,} 32$ Several of the energy transfer studies with BODIPY as the energy acceptor also include pyrene as the donor, which, however, was exclusively linked at the 1-position. ${ }^{31-44}$ The most widely used substitution pattern for connecting pyrene to BODIPY is the substitution at the boron centre. $^{31,33,34,36,38,40,41,45}$ Harriman et al. reported two simple triads, one consisting of a BODIPY dye with two pyrene chromophores connected to the BODIPY boron atom via an alkyne bridge and the other one being a mixed triad with pyrene and perylene as energy donors. Energy transfer has also been investigated by Ziessel et al. ${ }^{37}$ for species where pyrene is attached either directly or via bridges to the BODIPY at the methine carbon atom between the two pyrrole rings. By comparison of excitation and absorption spectra the authors estimated an effective energy transfer from pyrene to BODIPY in excess of $90 \%$ with a calculated energy transfer rate (by Förster theory) of $(1.5 \mathrm{ps})^{-1}$ when the pyrene is directly attached to the BODIPY, and $(27 \mathrm{ps})^{-1}$ when it is separated by an ethynylphenyl spacer. Vauthey et al. quite recently reported on a pyrene-BODIPY dyad in which the pyrene is attached at its 1-position via a phenylene spacer to the meso-position of the BODIPY dye. 
Here, excitation into the pyrene initiates energy transfer with $\tau=0.4$ ps to the BODIPY chromophore followed by internal conversion (IC) with $\tau<0.1 \mathrm{ps}^{44}$ No example of a dye conjugate where a pyrene is linked to the pyrrole units of a BODIPY chromophore is known to us. However, Burgess et al. $^{46}$ investigated the energy transfer in dyads consisting of BODIPY and anthracene with diverse substitution positions. They found efficient energy transfer in all compounds, but the energy transfer rate was fastest when the anthracene was attached at the 2-position of the BODIPY.

To shed light onto the dynamic light induced processes in the $\mathbf{P y}_{2} \mathbf{B}$ dye conjugate we perform here transient absorption (TA) measurements with fs time resolution to follow the ultrafast processes expected after excitation into the pyrene $S_{2}$ state. These experimental studies are supported by theoretical simulations of the light-induced nonadiabatic dynamics which have been performed in the frame of the field-induced surface hopping (FISH) method.

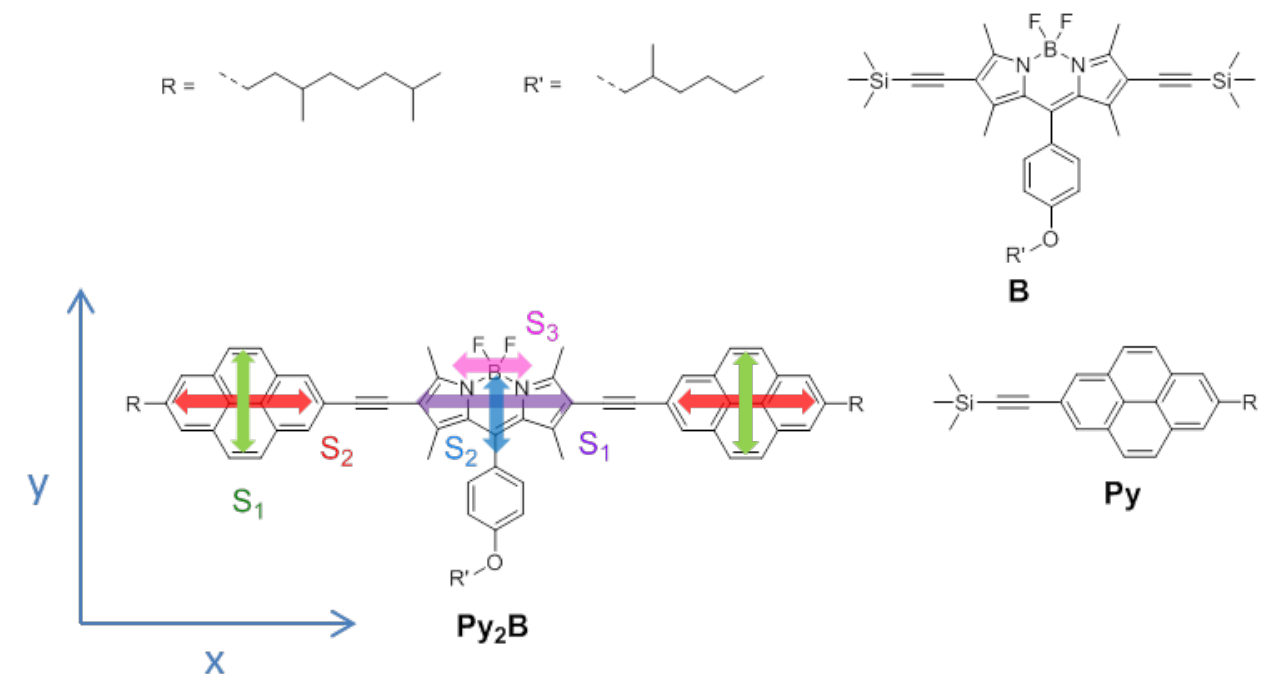

Fig. 1 Symmetrical pyrene-BODIPY-pyrene conjugate and its parent compounds. The inserted arrows mark the estimated chromophore-localised transition moment direction.

\section{Results and discussion}

The $\mathbf{P y}_{2} \mathbf{B}$ triad was synthesised by coupling the appropriate monomers via Sonogashira reactions. For detailed information see supporting information.

Steady state UV-vis absorption and fluorescence spectra. The triad absorption spectra were measured in dichloromethane (DCM) at room temperature and compared with those of its parent compounds $\mathbf{B}^{47}$ and the pyrene derivative Py (see Fig. 2). 
The absorption spectrum of $\mathbf{P y}_{2} \mathbf{B}$ covers a broad wavenumber range with one intense band originating from the BODIPY $S_{1} \leftarrow S_{0}$ excitation around $17200 \mathrm{~cm}^{-1}$. This band is clearly more diffuse ( $\mathrm{fwhm}=1790 \mathrm{~cm}^{-1}$ ) and also redshifted by $900 \mathrm{~cm}^{-1}$ than the one of the reference compound $\mathbf{B}$ ( $\mathrm{fwhm}=1080 \mathrm{~cm}^{-1}$ ). A weaker and broader band in the spectrum of $\mathbf{P y _ { 2 }} \mathbf{B}$ between 22000-27000 $\mathrm{cm}^{-1}$ stems from the overlapping BODIPY $S_{2} \leftarrow S_{0}$ and $S_{3} \leftarrow S_{0}$ excitation. ${ }^{30}$ The low anisotropy in the fluorescence excitation anisotropy measurements in poly-THF (see ESI) indicates that the $S_{2}$ state is polarised perpendicular to the $S_{1}$ state but the $S_{3}$ state is again polarised parallel to the $S_{1}$ state. At the blue edge of the spectrum the typical vibrational progression associated with the pyrene $S_{2} \leftarrow S_{0}$ absorption (see Fig. 2) is visible. The two BODIPY bands in $\mathbf{P y}_{2} \mathbf{B}$ are shifted to lower energy compared to the parent B chromophore. In contrast, the peaks of pyrene in $\mathbf{P y}_{2} \mathbf{B}$ remain at the same spectral position but are distinctly more diffuse and their intensities deviate from the expected doubled intensity of Py. This indicates that there is an additional band between 29000-33000 $\mathrm{cm}^{-1}$, which is caused by the interaction of the BODIPY dye and pyrene and which overlaps with the pyrene absorption peaks. The high anisotropy (ca. 0.33) in the fluorescence excitation anisotropy (see ESI) shows that the pyrene $S_{2}$ band and the overlapping band possess the same polarisation direction as that of the BODIPY $S_{1} \rightarrow S_{0}$ band, that is, polarisation along the long molecular axis $(x)$.

In the triad $\mathbf{P y}_{2} \mathbf{B}$ the shift and fwhm of the lowest energy BODIPY dye band indicate moderate interaction with the pyrene $\pi$-system in the ground and in the lowest excited state, which therefore appears to be largely localised at the BODIPY chromophore. This conclusion is drawn from the fact that the absorption features (band energies and band shapes) resemble those of the parent chromophores. On the other hand, exciton coupling theory predicts that even a strong coupling would lead to vanishing shifts of transitions in the coupled super-chromophore if the eigenstates of the monomer chromophores are energetically very far apart, that is, small shifts are no indication for the absence of electronic couplings. ${ }^{19}$ The squared transition moments of the lowest energy absorption band in $\mathbf{P y}_{\mathbf{2}} \mathbf{B}$ as determined by eq. 1 is only about $21 \%$ higher than that of the isolated parent dye $\mathbf{B}$ which also indicates that the original electronic character of this transition is retained in the ground state and Franck-Condon state in the triad (see Table 1).

$$
\mu_{\mathrm{abs}}^{2}=\frac{3 h c \varepsilon_{0} \ln 10}{2000 \pi^{2} N_{\mathrm{AV}}} \frac{9 n}{\left(n^{2}+2\right)^{2}} \int \frac{\varepsilon}{\widetilde{v}} d \tilde{v}
$$

a)

b) 

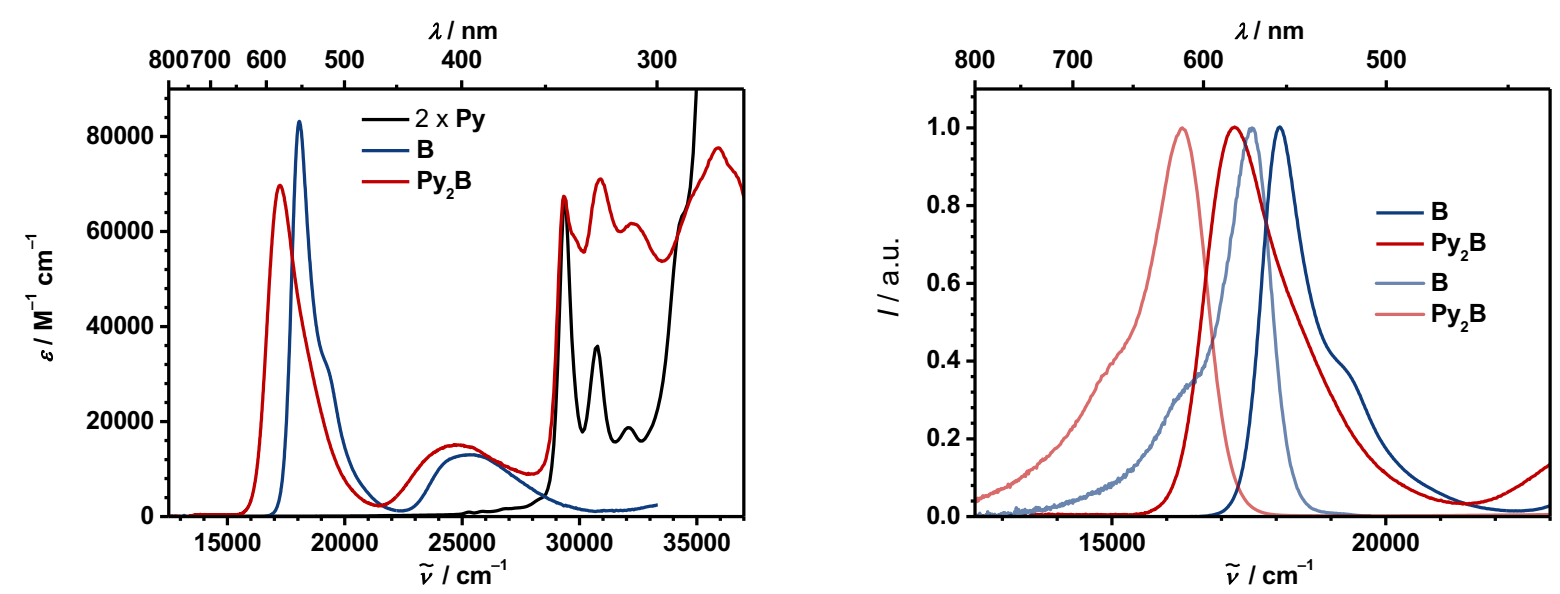

Fig. 2 (a) Absorption spectra and (b) normalised absorption and fluorescence spectra of $\mathbf{P y}_{2} \mathbf{B}, \mathbf{B}$ and $\mathbf{P y}$ in dichloromethane at r.t. For the emission spectra, the excitation of $\mathbf{P} \mathbf{y}_{2} \mathbf{B}$ was at $29400 \mathrm{~cm}^{-1}$. 
Table 1 Steady-state optical spectroscopic properties of the pyrene-BODIPY-pyrene triad and the corresponding parent compounds in dichloromethane at r.t.

\begin{tabular}{|c|c|c|c|c|c|}
\hline & $\begin{array}{c}\tilde{v}_{\mathrm{abs}} / \mathrm{cm}^{-1} \\
\left(\varepsilon / \mathrm{M}^{-1} \mathrm{~cm}^{-1}\right) \\
{\left[\mathrm{fwhm} / \mathrm{cm}^{-1}\right]}\end{array}$ & $\mu_{\mathrm{abs}}^{2} / \mathrm{D}^{2}$ & $\begin{array}{c}\tilde{v}_{\mathrm{fl}} \\
{\left[\mathrm{fwhm} / \mathrm{cm}^{-1}\right]}\end{array}$ & $\mu_{\mathrm{fl}}^{2} / \mathrm{D}^{2}$ & $\begin{array}{c}\phi_{\mathrm{fl}} \\
\left(\tilde{v}_{\mathrm{ex}} / \mathrm{cm}^{-1}\right)\end{array}$ \\
\hline Py & $\begin{array}{c}29400 \\
(33200)\end{array}$ & $\begin{array}{l}0.98 \text { (S1) } \\
12.6(\mathrm{~S} 2)\end{array}$ & 25180 & 26.2 & 0.10 \\
\hline$B^{47}$ & $\begin{array}{c}18100 \\
(83200)[1080]\end{array}$ & 48.4 & $\begin{array}{l}17600 \\
{[1030]}\end{array}$ & 44.1 & 0.84 \\
\hline $\mathrm{Py}_{2} \mathrm{~B}$ & $\begin{array}{c}17200 \\
(69600)[1790]\end{array}$ & 58.7 & $\begin{array}{l}16300 \\
{[1350]}\end{array}$ & 79.3 & $\begin{array}{c}0.72 \\
(18900)\end{array}$ \\
\hline
\end{tabular}

Direct excitation of a $\mathbf{P y}_{2} \mathbf{B}$ solution in DCM into the pyrene $\mathrm{S}_{2} \leftarrow \mathrm{S}_{0}$ absorption band at 29400 $\mathrm{cm}^{-1}$ results in strong BODIPY type fluorescence at $16300 \mathrm{~cm}^{-1}$ (see Fig. 2) which has a Stokes shift of $900 \mathrm{~cm}^{-1}$. This BODIPY emission of $\mathrm{Py}_{2} \mathbf{B}$ has a more pronounced structure with a smaller fwhm $\left(1350 \mathrm{~cm}^{-1}\right)$ whereas the absorption spectrum is distinctly more diffuse $\left(\mathrm{fwhm}=1790 \mathrm{~cm}^{-1}\right)$. This hints towards a more flexible ground state geometry with a flat hypersurface (possibly rotation around the $\mathrm{CC}$ triple bonds) than in the excited state where all components of the triad are coplanar. ${ }^{48}$ The fluorescence quantum yield of $\mathbf{P y}_{2} \mathbf{B}\left(\phi_{\mathrm{fl}}=\right.$ $0.72)$ is slightly lower than that of $\mathbf{B}\left(\phi_{\mathrm{fl}}=0.84\right)$ beyond the experimental error of ca. $5 \%$.

Computed absorption spectra and electronic states of $\mathrm{Py}_{2} \mathrm{~B}$. In order to simulate the thermally broadened absorption spectrum of $\mathbf{P y}_{2} \mathbf{B}$ we sampled the ground state canonical harmonic Wigner distribution at $\mathrm{T}=300 \mathrm{~K}$. Overall, 300 normal mode displacements and momenta were generated and converted back to Cartesian coordinates. The electronic absorption spectra for each individual structure from the ensemble have been calculated at the AM1-CIS and TD-CAM-B3LYP levels of theory and all stick spectra have been convoluted by Lorentzians in order to generate the continous curve. The resulting thermally broadened absorption spectra are presented in Fig. 3 together with the experimental spectrum. The theoretical spectra at both levels reproduce well the experimental absorption bands. In particular, the splitting between the first and the third absorption band is in excellent agreement and the second band is much broader than the first one, also in agreement with the experiment. 


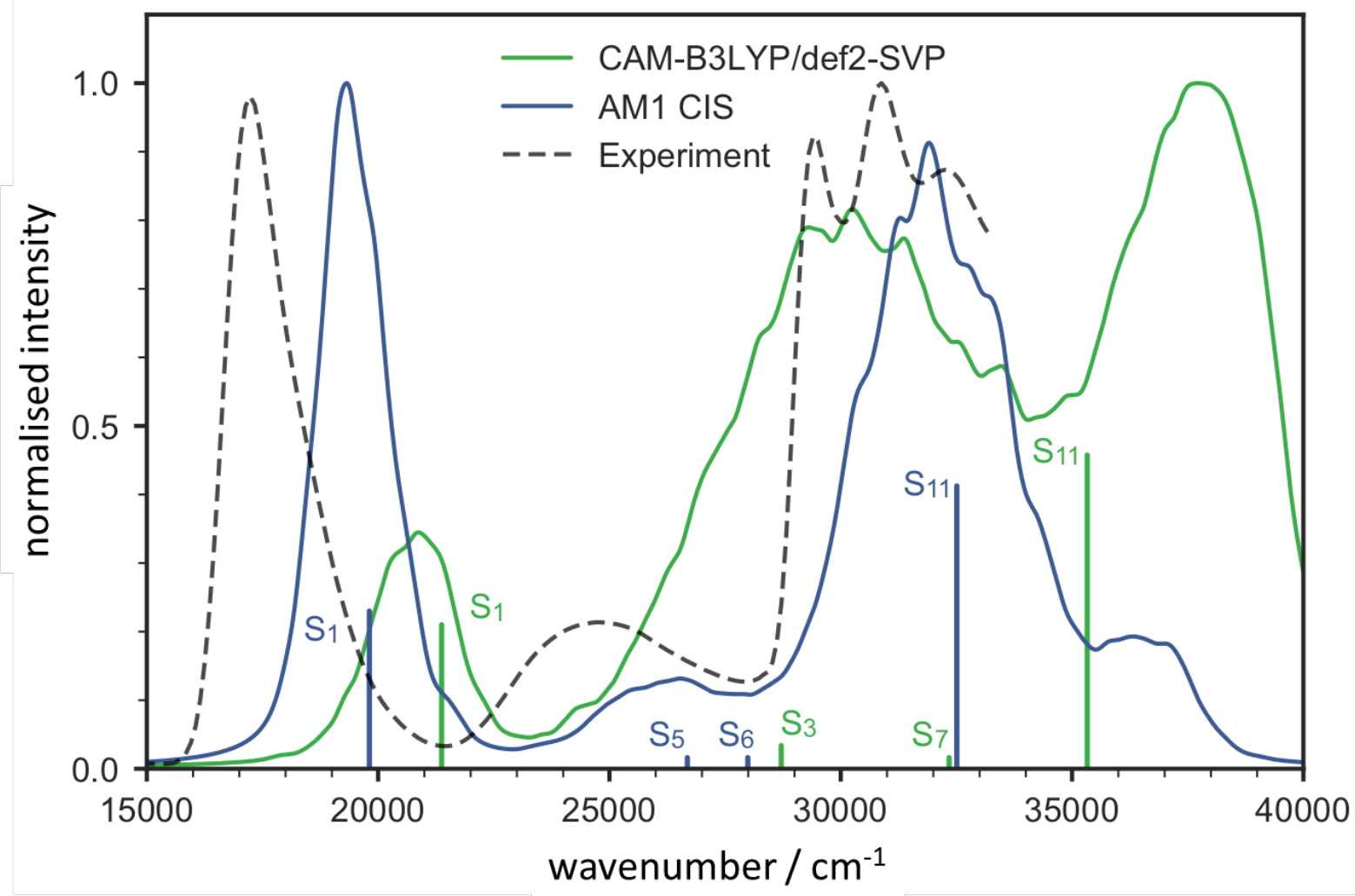

Fig 3. Comparison between the simulated spectra at the level of $A M 1 \mathrm{CIS}$ and TD-CAMB3LYP/def2SVP and experimental absorption spectrum of $\mathrm{Py}_{2} \mathbf{B}$. The vertical transition from the ground state are shown as sticks with the notation of the corresponding excited state. The simulated individual transitions have been convolved by a Lorentzian function with a width of $500 \mathrm{~cm}^{-1}$.

In Fig. 4 we present transition densities and natural transition orbitals of the dominant optically active electronic excited states calculated at the AM1-CIS level, which will be used to carry out nonadiabatic dynamics simulations, and at the TD-CAM-B3LYP level of theory. The character of the dominant electronic transitions is in perfect agreement between both employed methods. In the following, we will discuss the AM1-CIS results while the detailed comparison between the electronic states at the AM1-CIS and TDDFT level is given in the supporting information. As can be seen from Fig $4 a$ the $S_{1}$ state is mainly localised on the BODIPY-unit and its transition moment is oriented along the molecular longitudinal axis. The $S_{5}$ and $S_{6}$ states have a much weaker oscillator strength. The $S_{5}$ exhibits a strong localisation on the pyrene unit while the $S_{6}$ is partly localised on the BODIPY-unit. The second intense absorption peak at around $32000 \mathrm{~cm}^{-1}$ is dominated by the $\mathrm{S}_{11}$-state, which is delocalised over the whole system. The delocalisation arises due to the "J-type" coupling between the longitudinally polarised pyrene-states and the corresponding BODIPY-state. In the case of transversally polarised pyrene transitions the states remain uncoupled and are thus fully localised on the pyrene unit (cf. dark $S_{2}, S_{3}, S_{9}$, and $S_{10}$-states in between the bright ones in 
Fig. S3 and S4). These findings are in very good agreement with the qualitative picture obtained from the experimental absorption spectra.

In order to investigate the presence of charger-transfer (CT)-states and the composition of the excited states we have partitioned the transition densities into natural transition orbitals (NTOs, i.e. orbital representation which diagonalizes the transition density matrix). In the $S_{1}$ and $S_{2}$-state the electrons will be excited from a delocalised configuration to the one localised at the BODIPY-unit. These two excitations only differ in the sign of the hole so that in the case of the $S_{1}$ this leads to constructive overlap with particle but in the $S_{2}$ the overlap is almost destructive and only a small transition moment remains (see $\mathrm{SI}$ ). The $\mathrm{S}_{5}$-state is composed of two kinds of transitions and each consists of two different combinations of particles and holes.

Of course the combination of three chromophores leads to deviations of the electronic character of the triad excited states from those of the individual dyes because states with mixed character are generated by configuration interaction. Thus, the AM1-CIS computed $\mathrm{S}_{1}$ state clearly refers to the BODIPY $S_{1} \leftarrow S_{0}$ excitation around $17200 \mathrm{~cm}^{-1}$, the dark AM1 $S_{2}-S_{4}$ states and the bright $S_{6}$ state are y-polarised combinations of the (two) pyrene $S_{1}$ and BODIPY $S_{2}$ states. The AM1 $S_{5}$ state refers to an $x$-polarised combination of the two $x$ polarised pyrene $S_{2}$ states as is the very intense $A M 1 S_{11}$ state. 


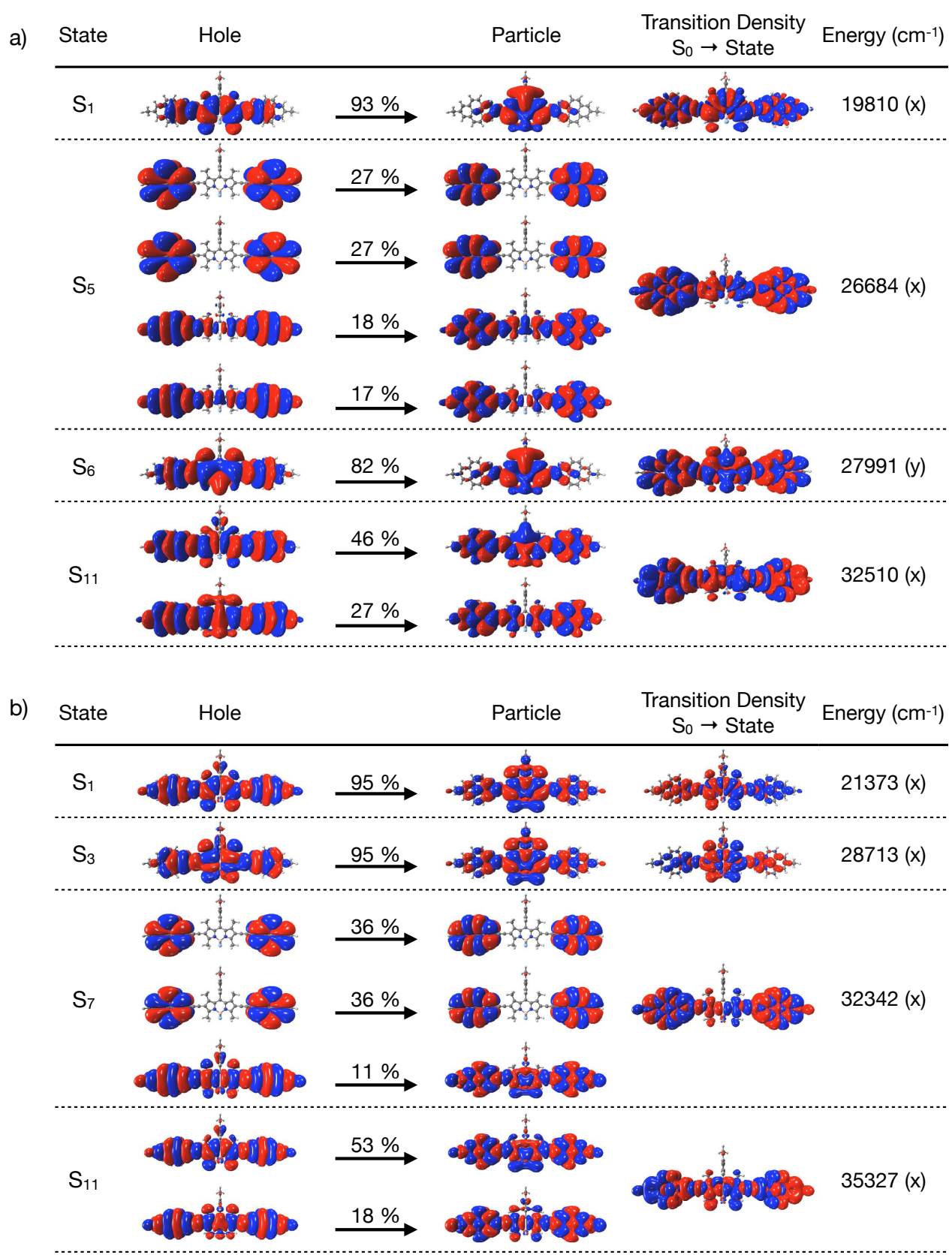

Fig 4. (a) Natural transition orbitals (NTOs) of the dominant excited states, the resulting transition densities (TDs), energies and the polarisation in braces at the AM1 CIS level. (b) NTOs of the dominant excited states, TDs and energies (polarisation) at the TDDFT level. Only contributions with eigenvalues $>0.1$ are shown.

Fluorescence lifetime measurements. The fluorescence lifetime of the triad was measured by time-correlated single photon counting (TCSPC, see Table 2) in DCM. When exciting the lowest energy band of the $\mathbf{P y} \mathbf{y}_{2} \mathbf{B}$ triad we measured a lifetime of $3.03 \mathrm{~ns}$, much shorter than 
that of B with $4.93 \mathrm{~ns}$. When excited at $31650 \mathrm{~cm}^{-1}$ at the pyrene $\mathrm{S}_{2}$ state the triad also shows fluorescence with the same lifetime as when excited at the lowest energy state of the BODIPY chromophore. Using these lifetimes and the fluorescence quantum yields the squared transition moment of the fluorescence $\mu_{\mathrm{fl}}{ }^{2}$ can be calculated via the Strickler-Berg equation: 49

$$
k_{\mathrm{fl}}=\frac{16 \cdot 10^{6} \pi^{3}}{3 h \varepsilon_{0}} \frac{n\left(n^{2}+2\right)^{2}}{9}\left\langle\tilde{v}_{\mathrm{fl}}^{-3}\right\rangle_{a v}^{-1} \mu_{\mathrm{fl}}^{2}
$$

where $\left\langle\tilde{v}_{\mathrm{fl}}^{-3}\right\rangle_{a v}^{-1}=\int I_{\mathrm{fl}} d \tilde{v} / \int I_{\mathrm{fl}} \tilde{v}^{-3} d \tilde{v}$ is the average cubic fluorescence energy and $k_{\mathrm{fl}}=\phi_{\mathrm{fl}} / \tau_{\mathrm{fl}}$ is the radiative rate constant. For the parent chromophore $\mathbf{B}$ the thereby evaluated (input from Table 1) fluorescence transition dipole moment is in reasonable agreement (within ca. $\pm 10 \%$ ) with those of the absorption spectra, which indicates no major changes of the electronic/geometrical nature in the lowest energy excited state. For the triad the situation is distinctly different, that is, the squared fluorescence transition moment is enhanced by ca. $35 \%$ compared to that of the absorption. This effect is even more impressing when comparing the squared fluorescence transition moments of $\mathbf{B}$ with that of $\mathbf{P y} \mathbf{y}_{2} \mathbf{B}$. Here the latter is $80 \%$ larger. A preliminary explanation for this observation is that the excited states of the triad undergo a structural rearrangement (possibly flattening) which results in an increased electronic coupling of the pyrene and BODIPY chromophore leading to an enhanced squared transition moment of the fluorescence process. ${ }^{47}$ This is also supported by the different band shapes of the BODIPY absorption in $\mathbf{P y} \mathbf{y}_{\mathbf{2}} \mathbf{B}$ and $\mathbf{B}$.

Table 2 Time resolved optical data from time correlated single photon counting (TCSPC) and transient absorption spectroscopy (TA) of the $\mathbf{P y}_{2} \mathbf{B}$ triad and reference compounds in dichloromethane at r.t.

\begin{tabular}{|c|c|c|}
\hline & $\begin{array}{c}\mathrm{Tf}_{\mathrm{f}} / \mathrm{ns}(\mathrm{TCSPC}) \\
{\left[\tilde{v}_{\mathrm{ex}} / \mathrm{cm}^{-1} / \widetilde{v}_{\mathrm{em}} / \mathrm{cm}^{-1}\right]}\end{array}$ & $\begin{array}{c}T / \mathrm{ps}(\mathrm{TA}) \\
{\left[\tilde{v}_{\mathrm{ex}} / \mathrm{cm}^{-1}\right]}\end{array}$ \\
\hline Py & $\begin{array}{c}34.8 \\
{[31650 / 25200]}\end{array}$ & \\
\hline B & $\begin{array}{c}4.93 \\
{[23920 / 17570]}\end{array}$ & \\
\hline $\mathrm{Py}_{2} \mathrm{~B}$ & $\begin{array}{c}3.03 \\
{[23920 / 16290]} \\
3.05 \\
{[31650 / 16290]}\end{array}$ & $\begin{array}{c}0.12 \\
1.6 \\
22 \\
460 \\
3100 \\
{[30800]}\end{array}$ \\
\hline
\end{tabular}


Femtosecond pump probe spectroscopy. The steady-state photophysical properties of the $\mathbf{P y}_{2} \mathbf{B}$ triad give first hints about the interactions in the pyrene-substituted BODIPY dye conjugate but no information about its photoinduced dynamics. Therefore, transient absorption (TA) measurements with fs time resolution were performed to distinguish between a dynamic energy transfer after excitation into the pyrene $S_{2}$ state or the formation of delocalised excitonic states with subsequent internal conversion processes. The symmetric triad was measured in DCM at r.t. and excited at the energy $\left(30800 \mathrm{~cm}^{-1}\right)$ of the 01-band of the pyrene $S_{2}$ state. This allows following the pyrene $S_{2}$ state dynamics by looking at the 00band without problems caused by excitation stray light. At this wavenumber, pyrene is primarily excited, but because of band overlap, a state that is formed by the interaction of pyrene with the BODIPY chromophore is excited, too. 

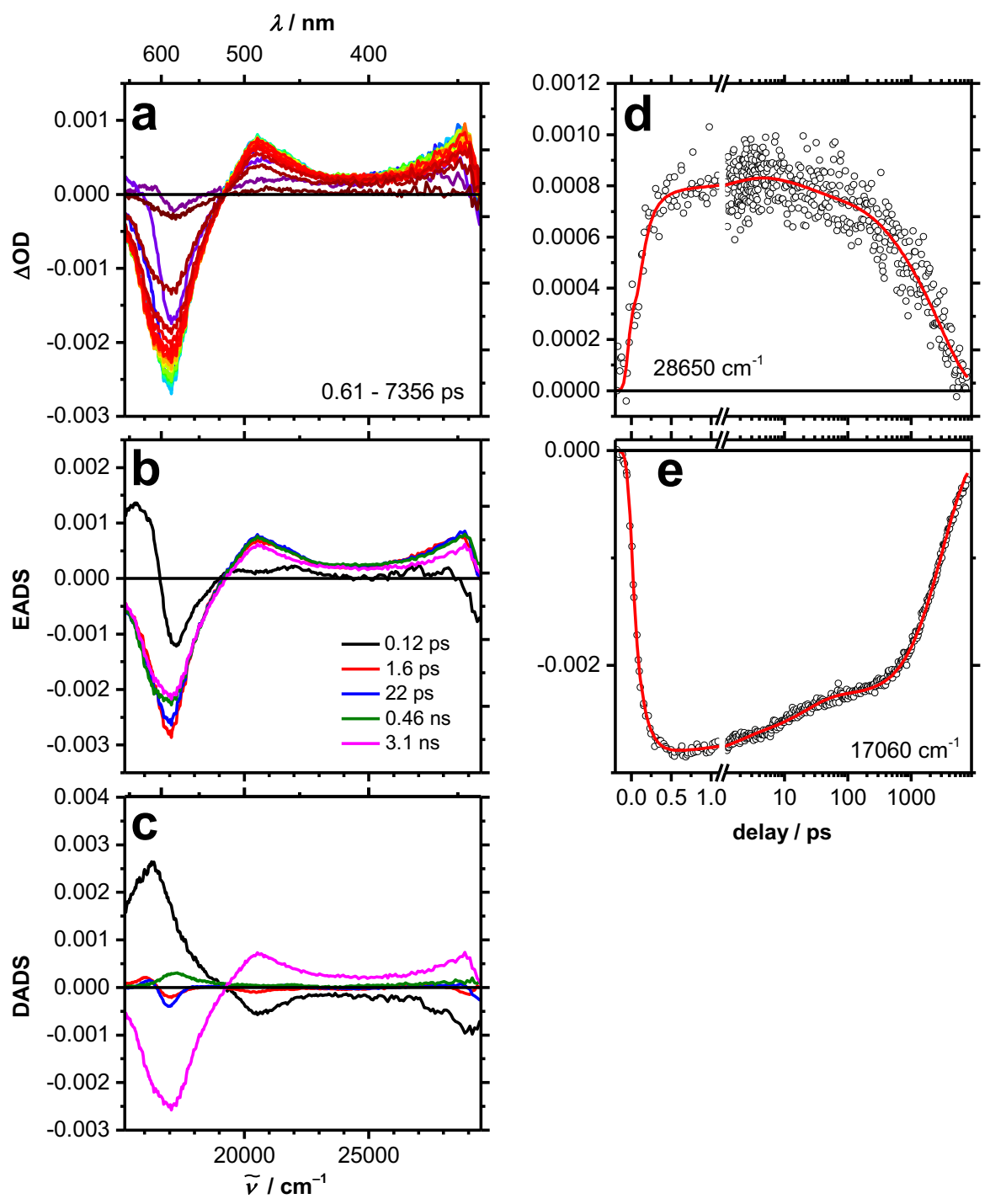

Fig. 5 Chirp corrected transient spectra of $\mathbf{P y}_{2} \mathbf{B}$ (a) in dichloromethane at $30800 \mathrm{~cm}^{-1}$ excitation. b) Evolution associated difference spectra from global fit of the TA data. c) Decay associated difference spectra from global fit of the TA data. d) and e) Transient decay profiles (black circles) at selected wavenumbers and global fit (red line). 
a)

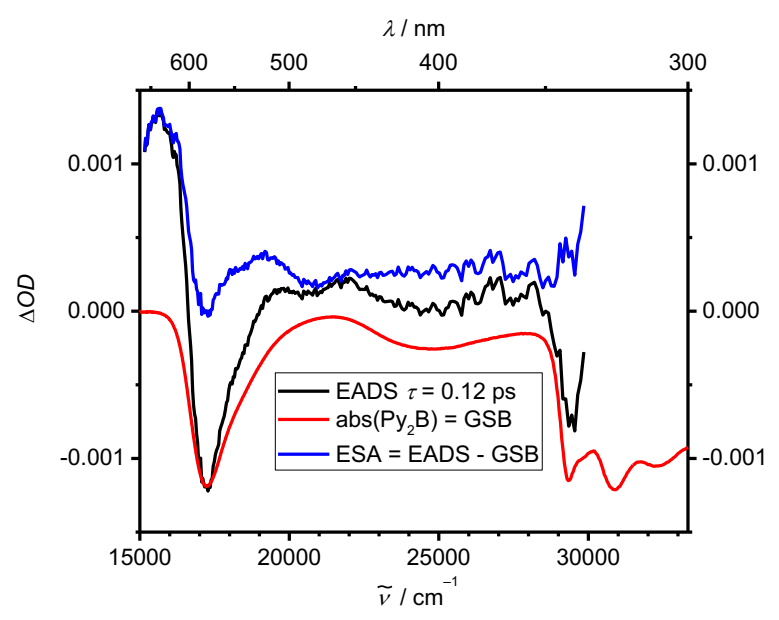

b)

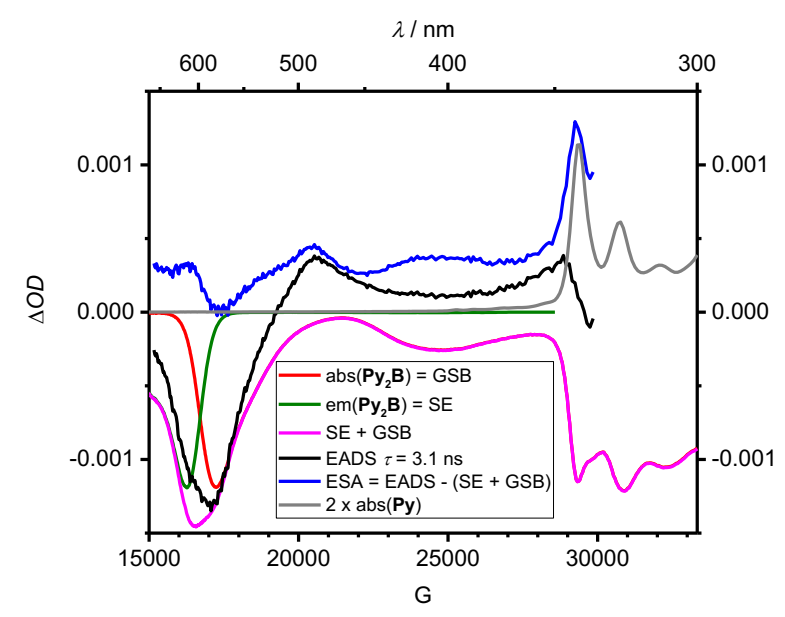

Fig. 6 Reconstruction of ESA spectra (in blue) of $\mathbf{P y}_{2} \mathbf{B}$ from EADS (a) with $\tau=0.12$ ps and (b) with $\tau=$ 3.1 ns using steady-state absorption and emission spectra.

Excitation of $\mathrm{Py}_{2} \mathrm{~B}$ at the 01-transition of the pyrene $\mathrm{S}_{2}$ state also excites a state resulting from interaction of pyrene and BODIPY significantly. The resulting TA spectra, which rise within instrument response (ca. $100 \mathrm{fs}$ ) show a strong ground state bleaching (GSB) below ca. $19000 \mathrm{~cm}^{-1}$ and excited state absorption (ESA) with the maxima at ca. 20500 and 28700 $\mathrm{cm}^{-1}$. Global analysis of the TA spectra with the minimum number of exponential functions gives decay associated difference spectra (DADS, amplitude spectra of species which evolve parallel in time) and the equivalent evolution associated difference spectra (EADS, spectra of species which evolve sequentially with increasing lifetimes) (Fig. 5) with $\tau=0.12,1.6,22$, 460 and $3100 \mathrm{ps}$. The first EADS with $\tau=0.12$ ps shows strong GSB at the spectral position of the lowest energy absorption of the BODIPY chromophore, but no concomitant stimulated emission (SE) at lower energy, and GSB at the 00-transition of the pyrene $S_{2}$ state at 29300 $\mathrm{cm}^{-1}$. This can also nicely be seen in comparison with the absorption spectrum of $\mathbf{P y}_{2} \mathbf{B}$ (red spectrum, see Fig. $6 \mathrm{a}$ ) and shows that the $\mathrm{Py}_{2} \mathbf{B}$ is excited and the resulting transition density is distributed over all triad constituents. However, this state is not the lowest energy state because otherwise one would expect SE arising at ca. $16000 \mathrm{~cm}^{-1}$. The EADS with longer time constants represent relaxation processes (see also DADS in Fig. 5c) and the last one with $\tau=3100 \mathrm{ps}$ ground state recovery is in excellent agreement with the TCSPC lifetime. Just for comparison, direct excitation of the lowest energy state of $\mathbf{P y}_{2} \mathbf{B}$ at $17200 \mathrm{~cm}^{-1}$ yielded very similar TA spectra and the global analysis gives similar EADS and time constants with exception of the ultrafast component, which is missing (see SI Fig. S2). We now compare the last EADS with $\tau=3100$ ps with the sum of emission and absorption 
spectra of $\mathrm{Py}_{2} \mathbf{B}$ yielding the ESA contribution (blue spectrum, see Fig. 6b). This constructed ESA shows a strong absorption peak at $29300 \mathrm{~cm}^{-1}$ which appears to be unreasonably strong. This peak indicates that using the triad absorption spectrum to simulate the GSB was not appropriate and that the transition density of the lowest energy excited state of $\mathbf{P y}_{2} \mathbf{B}$ is, on average, not entirely spread over the BODIPY and both pyrene chromophores, because otherwise one would expect that the absorption spectrum including all features of the triad constituents matches the GSB. In other words, localisation of excitation must have been occurred, at least to some extent. This somewhat conflicting result shall be elucidated by using computational methods, which allow looking on the electronic processes of a single molecule and not only on the average of an ensemble as in our experiments.

Light-induced nonadiabatic dynamics simulations. In order to investigate the dynamical processes induced in the $\mathbf{P y} \mathbf{y}_{2} \mathbf{B}$ triad after photoexcitation computationally, the nonradiative relaxation of the molecule has been simulated using the field-induced surface hopping (FISH) method ${ }^{50}$ that allows to treat the excitation by laser fields and nonadiabatic transitions on an equal footing.

The above described Wigner ensemble used to simulate the absorption spectrum has been also employed to generate 150 initial conditions for the FISH simulations. An ensemble of trajectories has been propagated for 500 fs in a manifold of 20 electronic states. For excitation a Gaussian shaped laser pulse resonant to the second broad absorption band has been used, whose full width at half maximum (FWHM) was $21 \mathrm{fs}$, and the field amplitude was maximal at $50 \mathrm{fs}$. The parameters were chosen to be similar to the experimental ones.

The resulting electronic state population dynamics is shown in Fig. 7 where we have grouped several excited states together. The laser pulse leads to significant population of the higher lying excited state. We have summed the population of the $S_{4}$ up to the $S_{20}$ state due to the fact that these states show a very similar population decay. Within a short time interval of about $50 \mathrm{fs}$, most of the trajectories leave the higher lying states and the $\mathrm{S}_{2}$ - and $\mathrm{S}_{3}$ states are populated (see green line in Fig. 7). However, the population of these states rises only up to $35 \%$, since some of the trajectories relax directly into the first excited state. The decay of the second and third excited states has a time constant of $97 \mathrm{fs}$ and leads to the population increase of the $S_{1}$ state with a very similar time constant of $99 \mathrm{fs}$. No transitions to the ground state take place within the simulation time, which is consistent with the experimentally observed fluorescence lifetime of ca. 3 ns. 


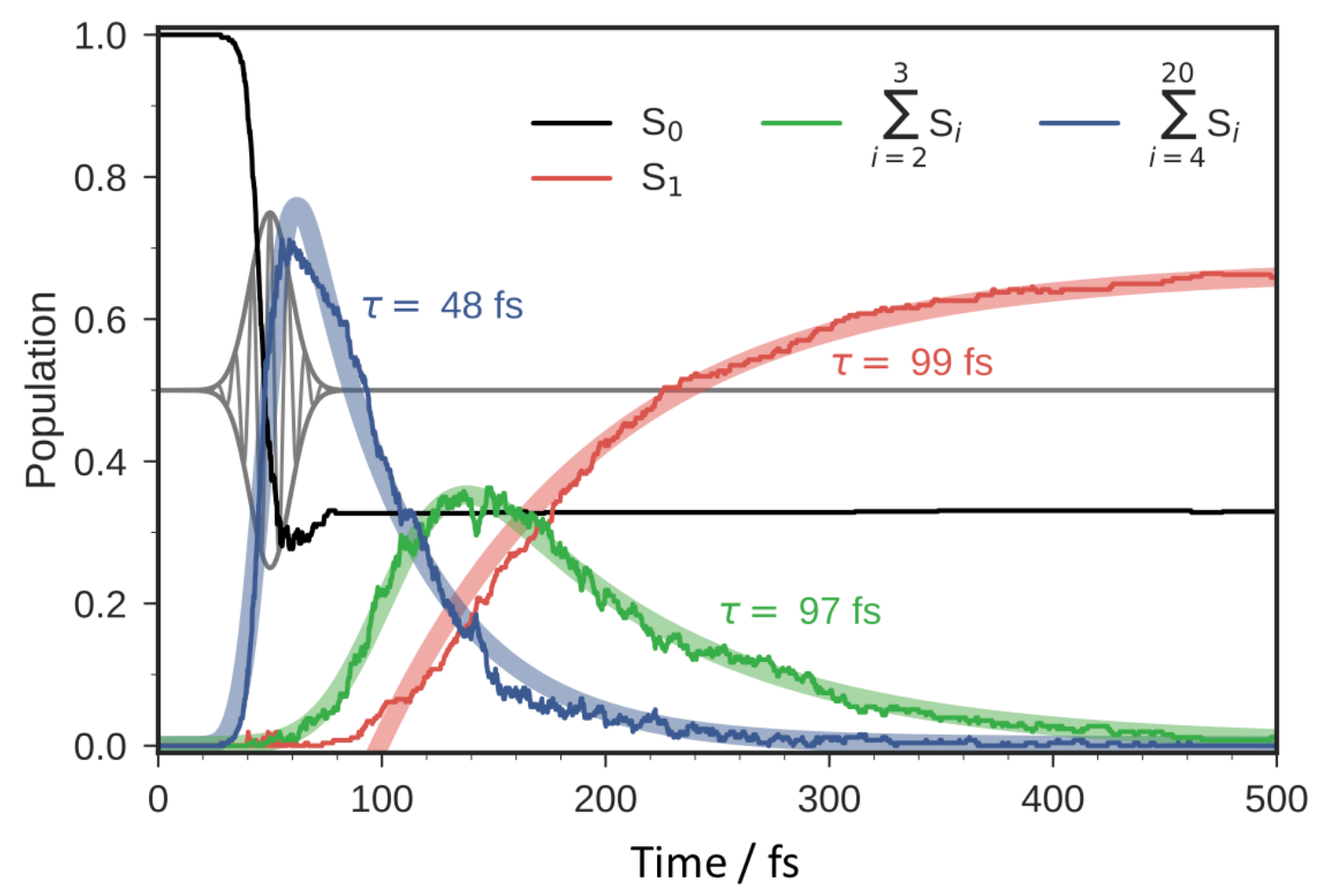

Fig. 7 Population of an ensemble of 150 trajectories of the electronic states during the field induced surface hopping dynamics of $\mathrm{Py}_{2} \mathrm{~B}$. The bold red line shows the fit of the $\mathrm{S}_{1}$ population to the following fit function: $F(t)=-A \exp \left(-\frac{t-t_{0}}{\tau}\right)+B$. The blue and green bold lines show the fit of the summed populations to the following function: $G(t)=A \exp \left(-\frac{t-t_{0}}{\tau}\right) \exp \left(\frac{b^{2}}{4 \sigma^{2}}\right)\left[1+\operatorname{erf}\left(\frac{t-t_{0}-\frac{b^{2}}{2 \sigma}}{b}\right)\right]$

In order to elucidate the time dependent orientation of the transition moment between the ground state and the actual state populated in the surface hopping simulations, we have performed an averaging at every time step over the whole ensemble of trajectories. After excitation with the laser pulse, a transition dipole moment of about $1.2 \mathrm{D}$ along the $\mathrm{x}$-axis to the actual state can be observed, which decays on a time scale less than 50 fs to $0.7 \mathrm{D}$ (see Fig. 8). During this time the dipole along the short molecular axis increases to about $0.3 \mathrm{D}$, with the maximum amplitude located at $80 \mathrm{fs}$. Within $150 \mathrm{fs}$ the transition moment along the $\mathrm{y}$-axis decreases to almost zero and the x-component moment increases up to $4.0 \mathrm{D}$.

The observed time-dependence of the transition dipole moment is in good agreement with experimentally obtained changes in the polarisation of the transition, which are shown on top of the state diagrams in Fig. 12 and refers to the intermediate population of the AM1-CIS computed $\mathrm{S}_{2}-\mathrm{S}_{4}$ and $\mathrm{S}_{6}$ state with y-polarisation. The time constant for the decrease in the longitudinal orientation with $35 \mathrm{fs}$ is of the same order of magnitude as the experimental constant for the switch from the longitudinal to the transversal polarisation ( $75 \mathrm{fs})$ of the $\mathrm{S}_{2}-\mathrm{S}_{1}$ internal conversion in pyrene (see Introduction). Furthermore, the subsequent increase in the 
longitudinal direction within 99 fs refers to the $S_{1}$ population and is in perfect accordance to the experimentally obtained lifetime, with a value of $120 \mathrm{fs}$, for step $\mathbf{A}$ in the state diagram that is rate determining for the corresponding process.

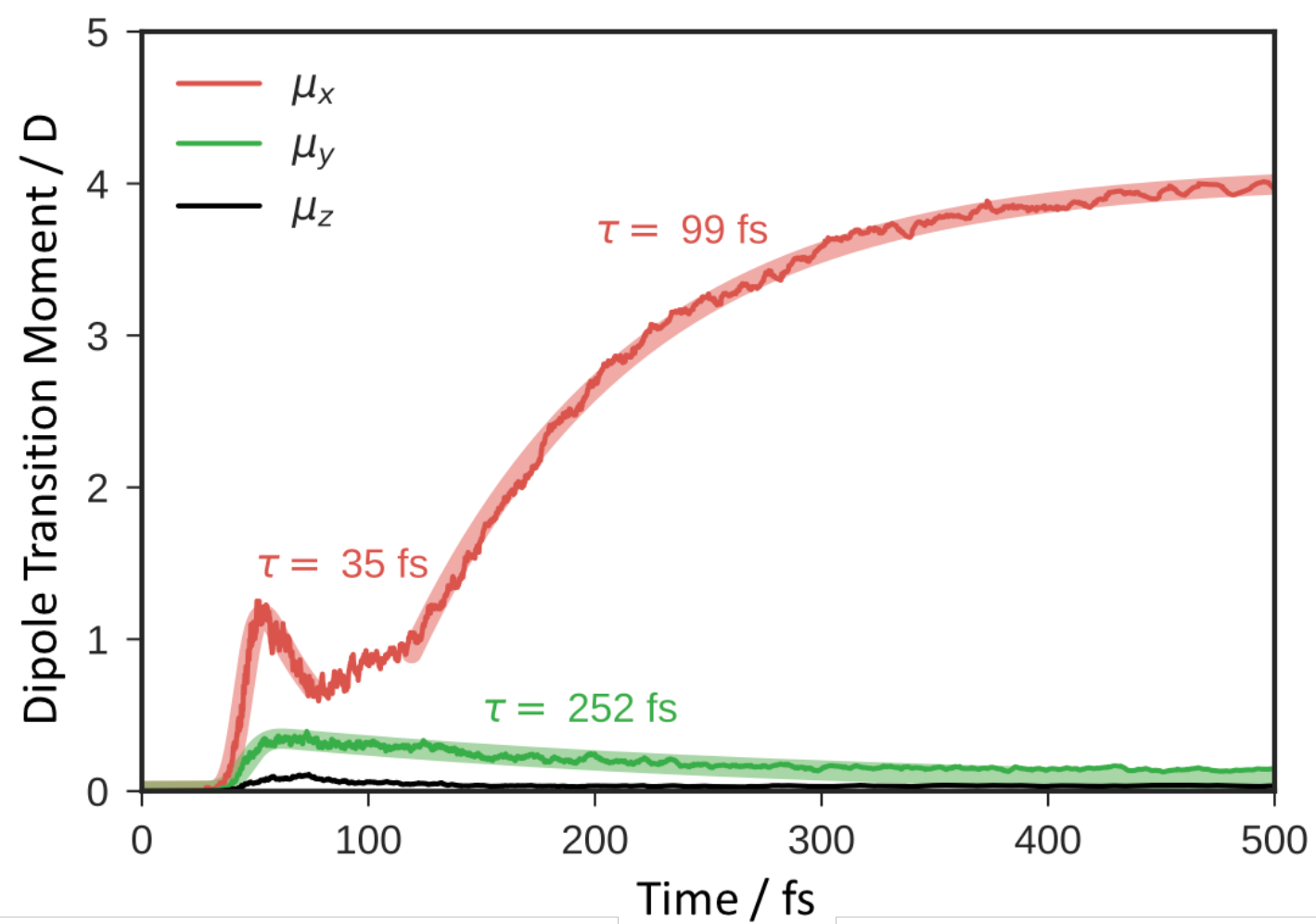

Fig. 8. Transition dipole moment of the actual populated electronic state of the whole ensemble. The $\mathrm{y}$-direction and the first $80 \mathrm{fs}$ of the $\mathrm{x}$-direction are fitted with the bold line to the following fit function: $G(t)=A \exp \left(-\frac{t-t_{0}}{\tau}\right) \exp \left(\frac{b^{2}}{4 \sigma^{2}}\right)\left[1+\operatorname{erf}\left(\frac{t-t_{0}-\frac{b^{2}}{2 \sigma}}{b}\right)\right]$. The bold red line of the x-direction between $120 \mathrm{fs}$ and 500 fs shows the fit to $F(t)=-A \exp \left(-\frac{t-t_{0}}{\tau}\right)+B$.

Analysis of the exciton localisation dynamics. In order to analyse the exciton dynamics, the adiabatic electronic states along the trajectories have been decomposed into the diabatic basis with excitations localised on individual monomers. We have monitored the population of these diabatic states along the trajectories by integrating the square of the electronic transition density (TD) for the adiabatic state which is populated at the given time step over the volume corresponding to each subunit and normalised the square of the whole transition density. This delivers two functions presented in Fig 9a for two representative trajectories (see also Fig. 10 for a pictorial representation) as well as the average of both functions over the whole ensemble which is presented in Fig. 9b. The value of 1.0 corresponds to the 
complete exciton localisation on the respective unit, while for the value of 0.5 , the TD is equally distributed over the whole system. The excitation leads to a short transient exciton localisation-delocalisation dynamics over the whole molecular system and after 150 to $200 \mathrm{fs}$ the exciton is strongly localised on the BODIPY subunit and resides there. On an ensemble level a very short transient exciton localisation on the 4-alkoxyphenyl group is observed which is accompanied by a decay, resulting in a coherent delocalisation within $10 \mathrm{fs}$. This delocalisation lasts for more than $100 \mathrm{fs}$ and is followed by strong localisation on the BODIPY subunit within another $100 \mathrm{fs}$.
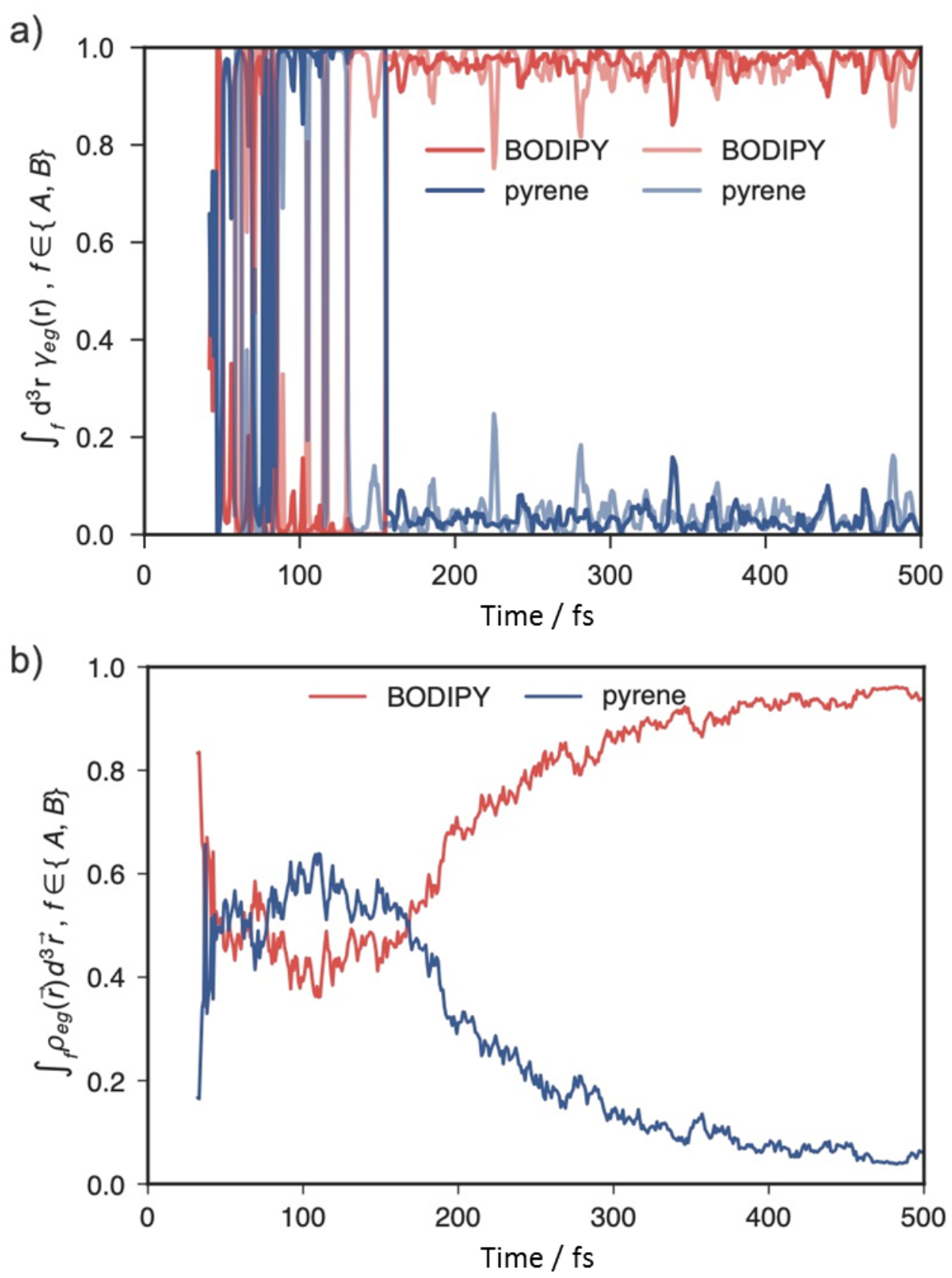

Fig 9. Integrated transition density on a given subunit (red, BODIPY; blue, pyrene units) for two single trajectories (a) and averaged over the whole ensemble (b). 
In order to gain a further insight into the localisation dynamics, we have calculated a 2D map of the transition densities between the electronic ground state and current state by integrating the transition density along the axis perpendicular to the molecular plane for the whole ensemble. The result is shown for selected time steps in Fig. 11 and the full animation is provided in the Supporting information. A superposition of the ensemble geometries at a given time step are shown in the bottom of each figure. The analysis of the exciton localisation shows a transient localisation/delocalisation within the first $60 \mathrm{fs}$ of the dynamics simulation on ensemble level. This is followed by a delocalisation over all subunits which lasts for around $100 \mathrm{fs}$. Afterwards the exciton localises on the BODIPY unit and remains there for the rest of the simulation time. During the time duration of the FISH simulations, the structures do not undergo any pronounced structural deformation but exhibit only relatively low amplitude vibrations.
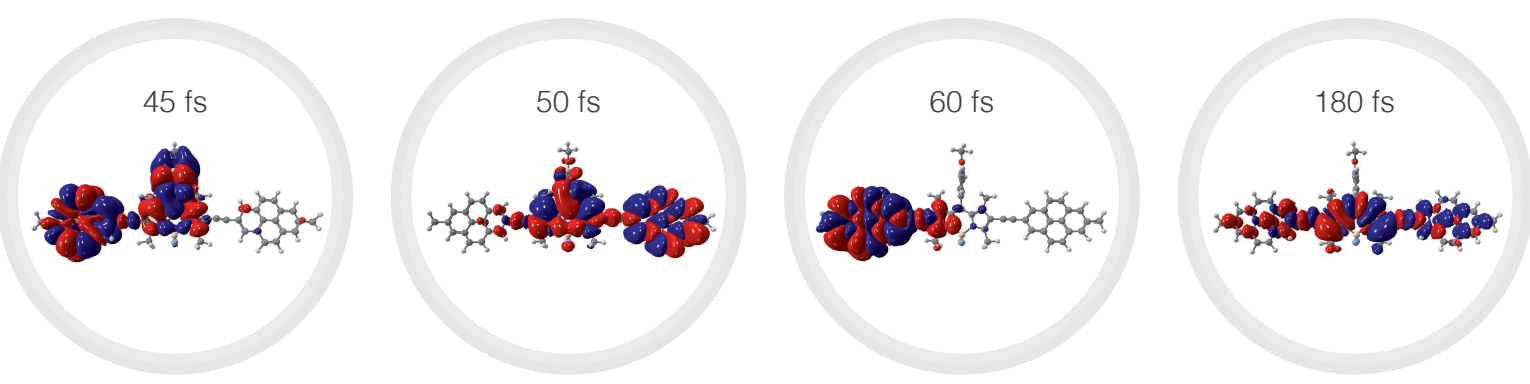

Fig 10. Transition densities between electronic ground state and current state on a single trajectory level. The depicted transition densities correspond to the integrated values shown in dark colours in Fig. 9a. 
a)
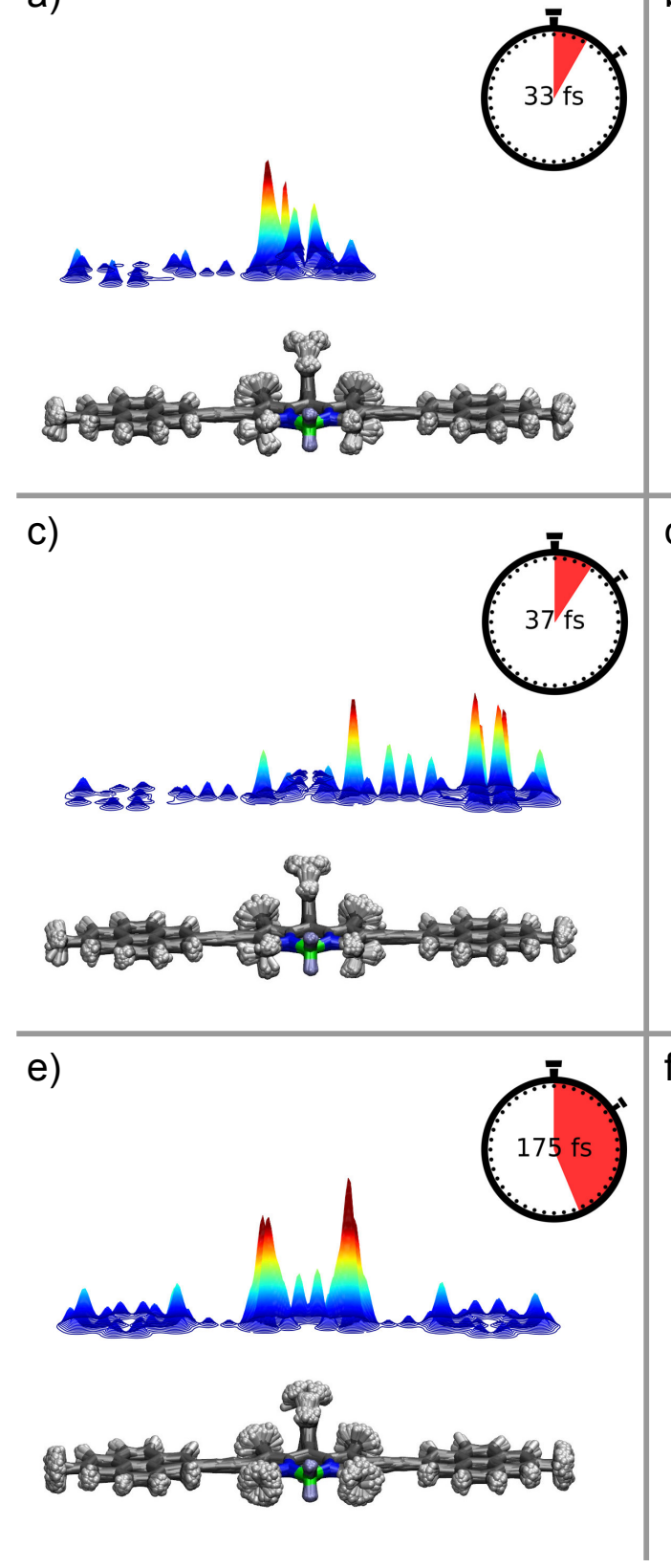

b)
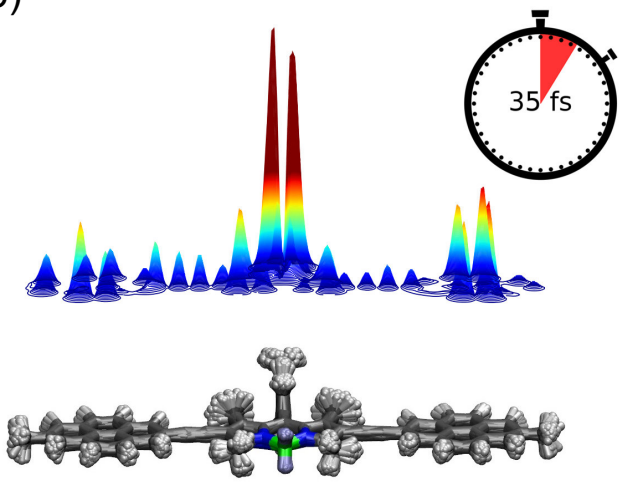

d)
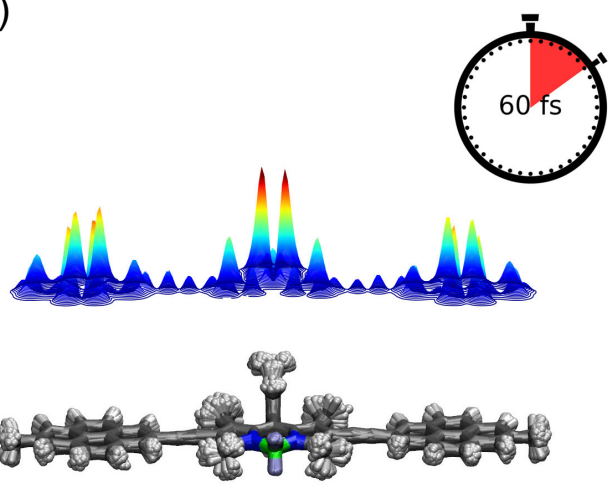

f)
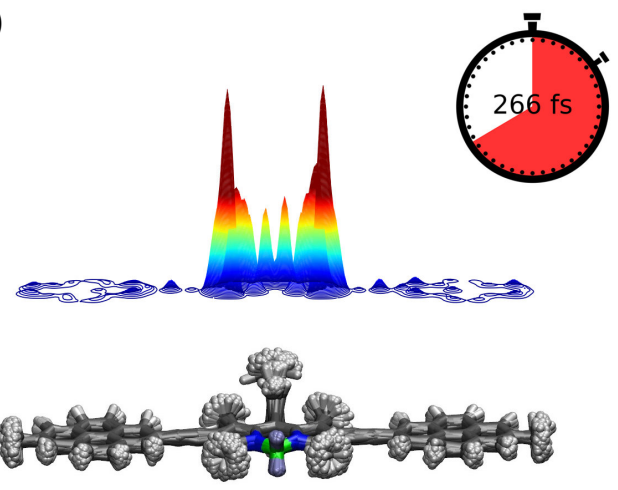

Fig 11. Transition densities between electronic ground state and current state after integration along the axis which is perpendicular to the molecular plane and averaging over the whole ensemble of trajectories (top). Superposition of the molecular structures at the given time frame (bottom). The numbers in the clock correspond to the simulation time (top right corner).

\section{Discussion and conclusion}



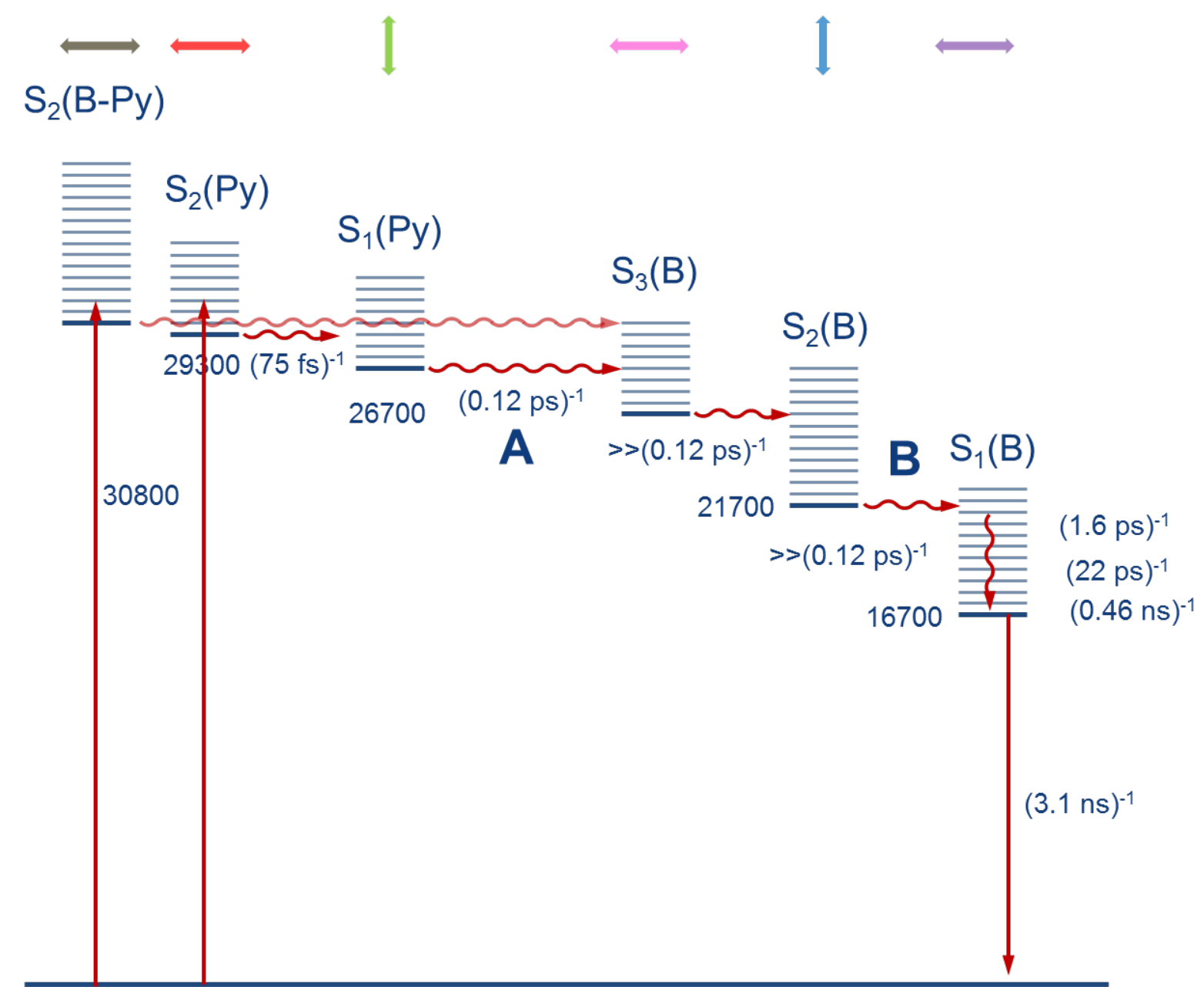

Fig. 12 State diagrams for the photophysical processes in $\mathrm{Py}_{2} \mathrm{~B}$. The assignment is made for the local chromophore states, as abbreviated in parentheses. The 00-state energies of the diverse states were estimated as follows: for $S_{2}(\mathrm{Py})$ from the most intense absorption peak. For $S_{1}(\mathrm{Py})$ from the highest energy peak of the fluorescence spectrum. For $S_{2}(B)$ from the intersection of a tangent to the lowest energy flank of the corresponding absorption spectrum with the $x$ axis. For $S_{1}(B)$ from the intersection of absorption and emission spectra. The energy of the partially delocalised $S_{2}$ (B-Py) states is estimated. For $\mathrm{Py}_{2} \mathrm{~B}$ the polarisation direction of each transition is given on top of the graph.

With the above collected information at hand we can construct a state diagram for the photoinduced processes in $\mathbf{P y} \mathbf{y}_{2} \mathbf{B}$ which is displayed in Fig. 12. At this point we stress that we assign the diverse states with labels, which describe the electronic character of the excited state best without any assumption whether we deal with a more localised or a more delocalised state that may also involve other molecular units than those indicated in the label.

From the TA experiments it appears that the rate determining step for the population of the lowest energy BODIPY state after excitation of the pyrene $S_{2}$ and concomitantly the B-Py state is process $A$ between the pyrene $S_{1}$ state and the BODIPY $S_{3}$ state with $\tau=0.12$ ps, 
assuming ultrafast IC between BODIPY $\mathrm{S}_{3}$ and $\mathrm{S}_{2} / \mathrm{S}_{1}$. The FISH simulations corroborate this interpretation as they predict a ca. $100 \mathrm{fs}$ time constant for the population of the $\mathrm{S}_{1}$ state from a state which has predominantly pyrene $S_{2}$ character, although this state is the AM1 $S_{2}$ state. However, from the time resolved experiments alone it is not quite clear whether we deal here with internal conversion within an excited superchromophore comprising the two pyrene units and the BODIPY dye or with energy transfer between one of the pyrene units and the BODIPY dye. The comparison of TA spectrum and steady-state absorption spectrum in Fig. 6a shows a very strong GSB at $29300 \mathrm{~cm}^{-1}$ comparable to the pyrene absorption at this energy and, at the same time, a pronounced GSB at the BODIPY absorption energy. This hints towards a sizable electronic coupling between the BODIPY and the pyrenes in the excited state although, the excited state has spectroscopically strong $S_{2}$ character of pyrene. While the former observation speaks for an IC process, the latter argues for an energy transfer process of almost decoupled chromophores. Likewise, in the final excited state (Fig. $6 b)$, the GSB at $29300 \mathrm{~cm}^{-1}$ indicates an electronic coupling between the BODIPY excited state and pyrene but the intensity of the GSB is much less than one would expect.

Thus, phenomenologically it appears that we deal with an intermediate case where the electronic coupling is strong enough to allow excitation of the whole triad but weak enough to retain most of the spectroscopic character of either BODIPY or pyrene states.

The latter may be due to the fact that the $S_{2}$ transition moment is oriented along the long axis of pyrene thus minimising excitonic interactions with the nearby lying $\mathrm{S}_{3} / \mathrm{S}_{2}$ states of BODIPY or squaraine.

As we have recently shown in a quantum-dynamical study in case of a dyad built up from two different squaraine dyes, the transition density of an individual molecule not only hops between the two excitonically coupled $S_{1} / S_{2}$ states but also between the two squaraine chromophores within the first several $100 \mathrm{fs}$ after excitation into $\mathrm{S}_{2}$. Thus, a site energy difference between the dyad chromophore constituents may lead to a dynamic behaviour but finally to localisation of excitation in the lower energy chromophore. This situation may also apply to the $\mathbf{P y}_{2} \mathbf{B}$ triad here, that is, fast hopping of transition density between BODIPY and pyrene may lead to a macroscopic ensemble in which on average there is more BODIPY excited than pyrene. This would lead to a GSB signal which does not reflect the steady state absorption spectrum in intensity.

Again, the FISH simulations shed light onto the dynamics. Looking at a single trajectory tells us that after pyrene excitation the excitation density hops several times over the two pyrene units and the BODIPY chromophore until it gets trapped onto the BODIPY chromophore (Figs. 9a and 10). However, on averaging the transition density of many trajectories, and that 
is what we observe experimentally, it appears that initially delocalised triad state comprising all three components are populated until localisation of excitation at the BODIPY occurs after ca 200 fs (Figs. 9b and 11). The latter process agrees excellently with the experimentally observed $120 \mathrm{fs}$ for the population of $\mathrm{S}_{1}$.

Thus, excitation of $\mathrm{Py}_{2} \mathbf{B}$ may be best described by neither IC nor energy transfer but by what we would like to call "dynamic exciton localisation" which clearly represents the bridge between the both extreme scenarios ${ }^{51}$ and also may involve symmetry breaking. ${ }^{52-55}$ 


\section{Experimental}

The synthesis of the triad is described in the Supporting Information.

\section{Optical spectroscopy}

Steady-state absorption measurements were carried out in $1 \mathrm{~cm}$ quartz cuvettes from Hellma in dichloromethane (Uvasol $\circledast$ from Merck) at r.t. using a Jasco V670 spectrometer. The pure solvent was used as a reference.

Fluorescence spectra were measured in $1 \mathrm{~cm}$ quartz cuvettes with Uvasol $($ solvents from Merck using an Edinburgh Instruments FLS980 spectrometer. The dissolved samples were purged with argon for 15 min before the measurement.

Fluorescence quantum yields were determined using optically dense samples in an integrating sphere following the method of Bardeen et al. ${ }^{56}$ to correct for self-absorption.

Fluorescence lifetimes were measured by time-correlated single-photon counting (TCSPC) with the FLS980 spectrometer using pulsed laser diodes at $15200 \mathrm{~cm}^{-1}$ or $19650 \mathrm{~cm}^{-1}$ or a pulsed light emitting diode at $31250 \mathrm{~cm}^{-1}$ as excitation source. The instrumental response was determined with a scatterer solution consisting of colloidal silicon in deionised water. All spectra were recorded under magic angle conditions using a fast PMT detector ( $\mathrm{H} 10720)$. Lifetimes were determined by deconvolution of the experimental decay (4096 channels) with the instrument response function and by fitting the decay curves with an exponential decay function using the FAST software (version 3.4.2).

\section{Femtosecond transient absorption spectroscopy}

All experiments were performed in quartz cuvettes from Spectrocell (Oreland, PA) with an optical path length of $0.2 \mathrm{~mm}$ equipped at r.t. All samples were dissolved in DCM, filtered and degassed for $30 \mathrm{~min}$. The optical density was adjusted to ca. 0.2 at the corresponding excitation wavenumber. The transient absorption spectra were performed with a NewportSpectra-Physics Solstice one-box amplified ultrafast Ti:Sapphire laser system with a fundamental wavenumber of $12500 \mathrm{~cm}^{-1}(800 \mathrm{~nm})$, a pulse length of $100 \mathrm{fs}$ and a repetition rate of $1 \mathrm{kHz}$

The output beam from the Solstice amplifier was split into two parts. One part was focussed onto a vertically oscillating $\mathrm{CaF}_{2}$ crystal to produce a white light continuum between $11800 \mathrm{~cm}^{-1}(850 \mathrm{~nm})$ and $28600 \mathrm{~cm}^{-1}(350 \mathrm{~nm})$. The resulting beam, which was polarised horizontally was used as the probe pulse. The second pulse was used to pump an optical parametric amplifier (TOPAS-C) from Light Conversion to generate the pump pulse with a pulse length of $140 \mathrm{fs}$ at the appropriate excitation wavelength. By using a wire grid (Moxtek) the polarisation axis of the pump pulse was set to magic angle relative to the probe pulse. 
The pump pulse (50 nJ, $\varnothing$ ca. $0.4 \mathrm{~mm}$ ) and the probe pulse $(\varnothing$ ca. $0.1 \mathrm{~mm}$ ) met at an angle of $6^{\circ}$ vertically in the cuvette. The probe pulse was measured by means of an CMOS sensor (Ultrafast Systems, Helios) in the range of $11900 \mathrm{~cm}^{-1}(840 \mathrm{~nm})$ to $29400 \mathrm{~cm}^{-1}(340 \mathrm{~nm})$ with an intrinsic resolution von $1.5 \mathrm{~nm}$. To compensate for white light intensity fluctuations, a reference beam was split off and detected with an identical spectrograph. Every second probe pulse was blocked by a mechanical Chopper $(500 \mathrm{~Hz})$ to measure the ratio of $I$ and $I_{0}$.

The computer-controlled stage (retro reflector in double pass setup) set the time difference between pump and probe pulse in 20 fs intervals from 0 fs to 4 ps and 4 ps to 8 ns in logarithmic steps with a maximum of 200 ps.

Before data analysis, the raw transient data were corrected for stray light and white light dispersion (chirp). The chirp was corrected by fitting a polynomial to the cross phase modulation signal of the pure solvent under otherwise experimental conditions. The evolution associated difference spectra (EADS) and the species associated difference spectra (SADS) were obtained from the corrected data by a global analysis using GLOTARAN ${ }^{57,58}$ software.

\section{Computational Details}

For the simulation of absorption spectra of the studied system, the structure has been first optimised in the frame of the semiempirical AM1 parametrisation ${ }^{59,60}$ and we calculated the vibrational modes to sample 150 structures from a phase space Wigner distribution at $300 \mathrm{~K}$. The excited states properties have been calculated in the framework of parametrized Configuration Interaction Singles (CIS). After performing extensive benchmark calculations on the energetics and characters of the excited states on the considered energy range, we have chosen an active space of 15 occupied and 15 virtual orbitals. The calculated stick absorption spectra were convolved with a Lorentzian function in order to obtain better comparability with the experimental data. Furthermore, to validate the applicability of AM1 for the studied system, we have also calculated the optical absorption spectra, natural transition orbitals $^{61}$ and transition densities at the TDDFT level, using the long-range corrected CAMB3LYP ${ }^{62}$ functional and the def2-SVP ${ }^{63}$ basis set as implemented in the Gaussian16 quantum chemical software package ${ }^{64}$ (see SI).

The light-induced nonadiabatic dynamics simulations have been performed using the fieldinduced surface hopping (FISH) methodology. This allows for realistic modelling of the excitation process induced by an ultrashort laser pulse as well as the description of the nonradiative transitions which are mediated by the nonadiabatic couplings. ${ }^{50,65,66}$ The laser fields employed in the simulations were parametrised in the time domain as Gaussian pulses. The full width at half-maximum (fwhm) for all applied pulses was $21 \mathrm{fs}$, and the field amplitude was maximal at $50 \mathrm{fs}$. The parameters were chosen to be similar to the experimental ones. For this purpose, the nonadiabatic dynamics simulations were performed 
in the manifold of the electronic ground and up to 20 excited electronic states. The nuclear degrees of freedom were propagated by solving the classical Newtonian equations using the velocity Verlet algorithm ${ }^{67}$ with a time step of $0.1 \mathrm{fs}$ for a total number of 5000 steps.

\section{Acknowledgement}

We thank the Deutsche Forschungsgemeinschaft for support of this work within the Research Group FOR 1809 and the Bavarian Ministry of Education, Culture, Research, and the Fine Arts for Support within the SolTech consortium. MISR and RM acknowledge the financial support within the ERC Consolidator Grant "DYNAMO” (Grant Nr. 646737)

\section{References}

1 A. Harriman, Chem. Commun., 2015, 51, 11745-11756.

2 B. Albinsson and J. Mårtensson, Phys. Chem Chem. Phys., 2010, 12, 7338-7351.

3 V. May, Dalton T., 2009, 10086-10105.

4 S. Speiser, Chem. Rev., 1996, 96, 1953-1976.

5 F. Laquai, Y. S. Park, J. J. Kim and T. Basche, Macromol. Rapid Comm., 2009, 30, 1203-1231.

6 I. Hwang and G. D. Scholes, Chem. Mater., 2011, 23, 610-620.

7 T. Brixner, R. Hildner, J. Koehler, C. Lambert and F. Wuerthner, Adv. Energy Mater., 2017, 7.

8 J. L. Bredas, D. Beljonne, V. Coropceanu and J. Cornil, Chem. Rev., 2004, 104, 4971-5003.

9 E. G. McRae and M. Kasha, Physical Processes in Radiation Biology, Academic Press, New York, 1964.

10 F. C. Spano, Accounts Chem. Res., 2010, 43, 429-439.

11 G. D. Scholes and G. Rumbles, Nat. Mater., 2006, 5, 683-696.

12 C. J. Bardeen, in Annual Review of Physical Chemistry, Vol 65, eds. M. A. Johnson and T. J. Martinez, 2014.

13 L. V. Herbert van Amerongen, Rienk van Grondelle, Photosynthetic Excitons, World Scientific, Singapur, 2000.

14 O. Kuhn and S. Lochbrunner, in Quantum Efficiency in Complex Systems, Pt li: From Molecular Aggregates to Organic Solar Cells, eds. U. Wurfel, M. Thorwart and E. R. Weber, 2011.

15 J. Knoester, Proceedings of the International School of Physics "Enrico Fermi" Course, IOS Press, Amsterdam, 2002.

16 E. Collini and G. D. Scholes, Science, 2009, 323, 369-373.

17 A. Chenu and G. D. Scholes, in Annual Review of Physical Chemistry, Vol 66, eds. M. A. Johnson and T. J. Martinez, 2015.

18 M. I. S. Röhr, H. Marciniak, J. Hoche, M. H. Schreck, H. Ceymann, R. Mitric and C. Lambert, J. Phys. Chem. C, 2018, 122, 8082-8093.

19 C. Lambert, T. Scherpf, H. Ceymann, A. Schmiedel and M. Holzapfel, J. Am. Chem. Soc., 2015, 137, 3547-3557.

20 C. Lambert, J. Ehbets, D. Rausch and M. Steeger, J. Org. Chem., 2012, 77, 6147-6154.

21 J. Merz, J. Fink, A. Friedrich, I. Krummenacher, H. H. Al Mamari, S. Lorenzen, M. Haehnel, A. Eichhorn, M. Moos, M. Holzapfel, H. Braunschweig, C. Lambert, A. Steffen, L. Ji and T. B. Marder, Chem.-Eur. J., 2017, 23, 13164-13180. 
M. Raytchev, E. Pandurski, I. Buchvarov, C. Modrakowski and T. Fiebig, J. Phys. Chem. A, 2003, 107, 4592-4600.

23 N. Krebs, I. Pugliesi, J. Hauer and E. Riedle, New J. Phys., 2013, 15.

24 W. Rodriguez-Cordoba, C. A. Sierra, C. O. Puentes, P. M. Lahti and J. Peon, J. Phys. Chem. B, 2012, 116, 3490-3503.

25 T. M. Figueira-Duarte and K. Müllen, Chem. Rev., 2011, 111, 7260-7314.

26 A. G. Crawford, A. D. Dwyer, Z. Liu, A. Steffen, A. Beeby, L.-O. Pålsson, D. J. Tozer and T. B. Marder, J. Am. Chem. Soc., 2011, 133, 13349-13362.

27 A. G. Crawford, Z. Liu, I. A. I. Mkhalid, M.-H. Thibault, N. Schwarz, G. Alcaraz, A. Steffen, J. C. Collings, A. S. Batsanov, J. A. K. Howard and T. B. Marder, Chem.-Eur. J., 2012, 18, 5022-5035.

R. Ziessel, G. Ulrich and A. Harriman, New J. Chem., 2007, 31, 496-501.

J. Karolin, L. B. A. Johansson, L. Strandberg and T. Ny, J. Am. Chem. Soc., 1994, 116, 78017806.

H. L. Kee, C. Kirmaier, L. H. Yu, P. Thamyongkit, W. J. Youngblood, M. E. Calder, L. Ramos, B. C. Noll, D. F. Bocian, W. R. Scheidt, R. R. Birge, J. S. Lindsey and D. Holten, J. Phys. Chem. B, 2005, 109, 20433-20443.

R. Ziessel and A. Harriman, Chem. Commun., 2011, 47, 611-631.

R. Ziessel, G. Ulrich, A. Haefele and A. Harriman, J. Am. Chem. Soc., 2013, 135, 11330-11344. M. A. H. Alamiry, J. P. Hagon, A. Harriman, T. Bura and R. Ziessel, Chem. Sci., 2012, 3, 10411048.

A. Harriman, G. Izzet and R. Ziessel, J. Am. Chem. Soc., 2006, 128, 10868-10875.

A. Harriman, L. Mallon and R. Ziessel, Chem.-Eur. J., 2008, 14, 11461-11473.

A. Harriman, L. J. Mallon, S. Goeb, G. Ulrich and R. Ziessel, Chem.-Eur. J., 2009, 15, 45534564.

Eur. J., 2005, 11, 7366-7378.

C. Goze, G. Ulrich and R. Ziessel, Org. Lett., 2006, 8, 4444-4448.

G. Ulrich, S. Goeb, A. De Nicola, P. Retailleau and R. Ziessel, Synlett, 2007, 1517-1520.

L. Bonardi, G. Ulrich and R. Ziessel, Org. Lett., 2008, 10, 2183-2186.

43 D. Bai, A. C. Benniston, J. Hagon, H. Lemmetyinen, N. V. Tkachenko and R. W. Harrington, Phys. Chem Chem. Phys., 2013, 15, 9854-9861.

M. Fakis, J. S. Beckwith, K. Seintis, E. Martinou, C. Nancoz, N. Karakostas, I. Petsalakis, G. Pistolis and E. Vauthey, Phys. Chem Chem. Phys., 2018, 20, 837-849. 11720-11729.

J. Gierschner, J. Cornil and H. J. Egelhaaf, Adv. Mater., 2007, 19, 173-191.

S. J. Strickler and R. A. Berg, J. Chem. Phys., 1962, 37, 814-822.

R. Mitric, J. Petersen and V. Bonacic-Koutecky, Phys. Rev. A: At., Mol., Opt. Phys., 2009, 79, 053416.

51 C. Kaufmann, W. Kim, A. Nowak-Krol, Y. Hong, D. Kim and F. Würthner, J. Am. Chem. Soc., 2018, 140, 4253-4258.

52 J. S. Beckwith, A. Rosspeintner, G. Licari, M. Lunzer, B. Holzer, J. Frohlich and E. Vauthey, J. Phys. Chem. Lett., 2017, 8, 5878-5883.

53 E. Vauthey, Chemphyschem, 2012, 13, 2001-2011.

54 S. Amthor, C. Lambert, S. Dümmler, I. Fischer and J. Schelter, J. Phys. Chem. A, 2006, 110, 5204-5214.

55 H. Marciniak, N. Auerhammer, S. Ricker, A. Schmiedel, M. Holzapfel and C. Lambert, J. Phys. Chem. C, 2019, 123, 3426-3432. 
T.-S. Ahn, R. O. Al-Kaysi, A. M. Muller, K. M. Wentz and C. J. Bardeen, Rev. Sci. Instrum., 2007, 78, 086105.

57 I. H. M. van Stokkum, D. S. Larsen and R. van Grondelle, BBA-Bioenergetics, 2004, 1658, 262262.

58 J. J. Snellenburg, S. P. Laptenok, R. Seger, K. M. Mullen and I. H. M. van Stokkum, J. Stat. Softw., 2012, 49, 1-22.

59 J. J. P. Stewart, J. Comput. Chem., 1989, 10, 209-220.

60 J. J. P. Stewart, J. Comput. Chem., 1989, 10, 221-264.

61 R. L. Martin, J. Chem. Phys., 2003, 118, 4775-4777.

62 T. Yanai, D. P. Tew and N. C. Handy, Chem. Phys. Lett., 2004, 393, 51-57.

63 F. Weigend, M. Haser, H. Patzelt and R. Ahlrichs, Chem. Phys. Lett., 1998, 294, 143-152.

64 M. J. Frisch, G. W. Trucks, H. B. Schlegel, G. E. Scuseria, M. A. Robb, J. R. Cheeseman, G. Scalmani, V. Barone, G. A. Petersson, H. Nakatsuji, X. Li, M. Caricato, A. V. Marenich, J. Bloino, B. G. Janesko, R. Gomperts, B. Mennucci, H. P. Hratchian, J. V. Ortiz, A. F. Izmaylov, J. L. Sonnenberg, Williams, F. Ding, F. Lipparini, F. Egidi, J. Goings, B. Peng, A. Petrone, T. Henderson, D. Ranasinghe, V. G. Zakrzewski, J. Gao, N. Rega, G. Zheng, W. Liang, M. Hada, M. Ehara, K. Toyota, R. Fukuda, J. Hasegawa, M. Ishida, T. Nakajima, Y. Honda, O. Kitao, H. Nakai, T. Vreven, K. Throssell, J. A. Montgomery Jr., J. E. Peralta, F. Ogliaro, M. J. Bearpark, J. J. Heyd, E. N. Brothers, K. N. Kudin, V. N. Staroverov, T. A. Keith, R. Kobayashi, J. Normand, K. Raghavachari, A. P. Rendell, J. C. Burant, S. S. Iyengar, J. Tomasi, M. Cossi, J. M. Millam, M. Klene, C. Adamo, R. Cammi, J. W. Ochterski, R. L. Martin, K. Morokuma, O. Farkas, J. B. Foresman and D. J. Fox, Gaussian 16 Rev. B.01, Wallingford, CT, 2016.

65 P. G. Lisinetskaya and R. Mitric, Phys. Rev. A: At., Mol., Opt. Phys., 2011, 83, 033408.

66 M. I. S. Röhr, J. Petersen, M. Wohlgemuth, V. Bonacic-Koutecky and R. Mitric, Chemphyschem, 2013, 14, 1377-1386.

67 W. C. Swope, H. C. Andersen, P. H. Berens and K. R. Wilson, J. Chem. Phys., 1982, 76, 637649. 